\title{
Final Thoughts on Women in Sport Coaching: Fighting the War
}

\author{
Nicole M. LaVoi \\ University of Minnesota
}

\author{
Jennifer E. McGarry \\ University of Connecticut
}

\author{
Leslee A. Fisher \\ University of Tennessee
}

This collection of articles about and for women in sport coaching provides more evidence of the occupational landscape and experiences of women. As with countless empirical articles before, the eight articles in this special issue of Women in Sport and Physical Activity Journal (WSPAJ) further uncover and reveal that structural-level systemic bias is deeply embedded within the culture of sport-the data tell the story. With more data, the story plotline becomes sharply focused and illuminates the many obstacles women coaches face and how challenging it is to change the gendered system. As Pat Griffin (2015) argued, a war on women coaches exists and currently continues to rage. The war is exacerbated by

"misogyny, sexism, and homophobia. This trifecta of hostility towards women in athletics is made more threatening in an athletic climate in which financial resources are strained to the max and athletic administrators in schools large and small buy into the pipe dream of cultivating big time football (and men's basketball) as the salvation of cash strapped athletic departments." (Griffin, 2015)

To fight the war, data is warranted and must be systematically organized and leveraged to maximize change efforts.

In 2012, LaVoi and Dutove (2012) first outlined the Ecological Model of Barriers and Supports for Women Coaches, which was extended by LaVoi (2016) in Women and Sports Coaching to include intersectionality and power. The Ecological-Intersectional Model (EIM; LaVoi, 2016), derived from Bronfenbrenner's ecological systems theory, specifies that "human development and experience is influenced by a variety of proximally located individual, interpersonal, organizational, and socio-cultural environmental systems" (emphasis added; p. 19) across one's lifespan and career trajectory. The purpose of the EIM was to systematically organize existing literature, stimulate dialogue, identify gaps in the literature to help researchers target inquiry, and guide decision-makers to improve the occupational experiences for women coaches. What is clear from the data is that far more barriers than supports exist for women coaches - particularly for women with systematically marginalized identities (e.g., race, sexuality, age, class, ability, parenthood, and ethnicity; LaVoi, 2016). Ironically, the individuals in the system with the least power (i.e., women) often get blamed for the lack of women head coaches, a visible position of power.

Women are often blamed and held responsible, particularly by male athletics directors (Kane \& LaVoi, 2018), for the scarcity of

LaVoi is with the School of Kinesiology, University of Minnesota, Minneapolis, MN. McGarry is with the Department of Educational Leadership, University of Connecticut, Storrs, CT. Fisher is with the Department of Kinesiology, Recreation, and Sport Studies, University of Tennessee, Knoxville, TN. LaVoi (nmlavoi@umn. edu) is corresponding author. women in coaching due to their choices, failing to apply, lacking competencies or confidence, being less interested than men, focusing on family over career, or not taking job offers (LaVoi, 2016). Conversely, women athletics directors and senior woman administrators attributed the "good old boys" network and conscious and unconscious bias as the reasons for the scarcity of women coaches (Kane \& LaVoi, 2018). Blaming women ensures that the androcentric systemic status quo of male dominance remains intact. Feminist sport scholars over the decades have argued that male control of, and deeply embedded gendered power within, sport is a primary factor for the absence, scarcity, and stagnation of women in leadership positions. Unfortunately, little has changed in the occupational sport landscape for women over time, despite a plethora of research, advocacy, and action. As Norman, RankinWright, and Allison (2018) argued, the underrepresentation of women coaches must be reframed as "a symptom, or an outcome of a deeper issue, rather than the problem in itself" (p. 395). Fortunately, in the eight years since the EIM was proposed, researchers have used the model to focus efforts and further understanding that can potentially lead to social change, and the articles in this special issue are no exception.

\section{Locating the WSPAJ Special Issue Papers within the EIM}

Many contributions to the knowledge of barriers and supports in the EIM model for women coaches were evident in this special collection of papers. Cunningham et al. (2019) used a multilevel approach to highlight how occupational constraints limit individual women's aspirations and intentions to remain in coaching compared to male counterparts, and provided compelling evidence to assist in railing against common "blame the women" narratives for the lack of women in coaching. Women's professional choices are influenced by occupational and socio-cultural factors. Clarkson et al.'s (2019) piece reveals that gender is deeply embedded across the EIM model as a barrier to career development and progression for women coaches at all levels of competition from youth to elite. Clarkson's findings complement and reinforce what Cunningham et al. argued-gendered systems influence women's career trajectories and choices of women.

Two of the articles addressed career pathways into and through women's NCAA Division-I basketball. In Wasend and LaVoi's article (2019), the power of same- versus cross-sex role models, a possible interpersonal-level support in the EIM, was examined as an influence for female college athletes to enter into and stay in the coaching profession. The authors illuminated evidence for the power of same-sex role models for inspiration, aspiration, education, emulation, information, and access to a female network in helping early career women navigate and stay in coaching. 
Same-sex social support and role models matters. Larsen and Clayton (2019) used an intersectional lens of gender and race to examine career pathways of women's basketball head coaches, and found both play a significant role in influencing women's career trajectories. Larsen and Clayton's results add to the individual level in terms of intersectionality, and complemented and extended Cunningham et al. and Clarkson et al.'s findings that gender and racial bias permeates every level of the EIM and function as barriers to advancement and retention.

Multiple papers in this special issue outlined and evaluated programs targeted to develop women coaches. Allen and Reid (2019) and Culver et al. (2019) specified multifaceted programs, best practices, and lessons learned for developing women coaches in Scotland and Canada, respectively. Both papers add practical and applied knowledge of supports at the individual and interpersonal levels of the EIM model, that can be adapted to other contexts to help develop and retain women coaches and improve equitable representation of women leaders in sport. Krahn (2019) evaluated the effectiveness of Canadian sport policies, an organizational structural level variable in the EIM, that have potential to inform mentorship and sponsorship programs that help advance careers of high performance women coaches. Unfortunately, based on Krahn's analysis, policies fell short of intended outcomes of developing and supporting women coaches due to lack of practical strategies and the inclusion of specific language around mentorship and sponsorship. Research and data inclusive of the voices and experiences of women must inform policy development.

Banwell, Kerr, and Stirling's (2019) paper explicated the role of mentorship and sponsorship, which reside at the interpersonal level of the EIM, and the need to learn from non-sport domains. Sponsors (a majority of whom are men) have greater power in the system at the organizational level of the model, and, therefore, may be more effective in advancing the careers of women. Based on the data, Banwell argues women coaches need both male and female mentors over the entire career trajectory, and that mentorship must be supported at the organizational level. These data highlight both mentorship and sponsorship is necessary in helping women coaches overcome and navigate a highly gendered and discriminatory system.

Taken together, the papers in the special issue highlight the utility of locating where empirical efforts map into and through the EIM model, and in doing so revealed how different levels are intertwined and influence each other bi-directionally. Women coaches do not exist in a vacuum - their experiences are influenced by the people, organizational culture, and policies of those in power and the societies in which they live. To hold women responsible for the scarcity of women coaches is not only abundantly simplistic, but myopic.

\section{Gaps Remain in the Literature}

None of the papers in this special issue explicitly or directly examined socio-cultural level barriers or supports, collectively. However, the papers do reveal a highly gendered system which privileges men and functions to keep a male-dominated system in place. Despite being a global leader in civil rights for girls and women in sport due to Title IX, it appears that the United States lags behind other countries in terms of mentorship, programming, development, and organizational policies. The work being done in Canada and Western Europe will guide knowledge in how to develop women coaches and create an organizational climate where women of all identities feel safe, valued, and supported. Researchers must move away from barrier work and focus on what factors support women coaches and help them not only survive, but thrive.
In order to understand supports for women coaches, more light needs to be shed on individuals in power and decision-makers who are doing it "right"- such as gender allies. Allies are defined as members of dominant social groups who are critical members in the pursuit of meaningful change, and gender allies are a fruitful group that warrants examination (Heffernan, 2018). LaVoi and Wasend (2018) recently interviewed college athletics directors who employ and have hired a majority of women head coaches for their women's teams. They found this group of male and female allies to have an explicit, unapologetic belief that competent women existed, to have diligently and systematically recruited women, to have felt women should be coached by women, and to have intentionally created an organizational climate where women felt cared about, valued and supported. Additional research is needed on how to identify existing gender allies and on what processes are effective to develop more gender allies that in turn may change the occupational sport landscape. More ideas for a call to action and future research follow.

As Fink (2016) stated, "Sexism in sport is not tidy, it is a downright messy matter. The ideals of meritocracy and fair play embedded in sport make it difficult for people to believe that it provides advantages for some groups over others . . ." (p. 4). However, the papers in this special issue clearly confirm that the state of affairs for women coaches is not fair and not improving, and, in fact, "women are losing ground" (Banwell, Kerr, \& Stirling, 2019, p. 128). Despite the fact that many formal barriers have been removed through policies and legislation, the scholars in this issue have illuminated that implicit and entrenched bias remains (Clarkson, Cox, \& Thelwell, 2019; Fink, 2016). While it might appear that by simply increasing the numbers of women who enter coaching, and remain in coaching, we would arrive at a solution, an "adding women and stir" approach is not the answer and on its own is insufficient (Madsen \& McGarry, 2016), as we know that "it doesn't matter how successful you are, at some point people will still say something pretty down about you" (Allen \& Reed, 2019, p. 104). The job of a coach itself is stressful and challenging enough. For women coaches, additional challenges include low organizational support in the form of a lack of encouragement and validation, and less pay for similar, or even more, work. It begs the question, "Do I really have to deal with this on top of the pressures of the job?" (Clarkson, Cox, \& Thelwell, 2019, p. 78). Fink pointed to examples, like those women coaches face, of how sexism remains relatively "uncontested in sport" (Fink, 2016, p. 1). And, Shaw and Frisby (2006) stated that we have to critique such practices at the structural level that reflect socio-cultural, embedded beliefs - many of which are entirely implicit- that allow us to think we are treating all coaches equitably when we are not.

The bottom line is that women coaches' career mobility has been limited by embedded gender bias in organizational practices, and this bias has been used to scrutinize women coaches more than their male peers (Burton \& LaVoi, 2016; Clarkson, Cox, \& Thelwell, 2019). Krahn (2019) tells us that the lack of consistency between research, programming, and policy has contributed to a situation where the low numbers of women in sport coaching has been positioned as a women's issue rather than a systemic issue. The only way to contest that situation is through connecting research, programming, and policy, and doing so explicitly.

\section{Future Research and Call to Action: Everyone Can do Something!}

Moving forward, researchers first need to address the intersectional identities of women coaches (Banwell, Kerr, \& Stirling, 2019; 
Clarkson, Cox, \& Thelwell, 2019; LaVoi, 2016). Clarkson et al. (2019) calls for advancing the intersectional aspect of LaVoi's model, and one of Banwell et al.'s (2019) participants explicitly states that those in positions of power should be ". . . making sure young women, and not just young women, but women of other underserved minorities can see themselves in the leadership and as examples of excellence . .." (p. 132). Griffith (2015) pointed out the trifecta of hostility toward women in sports which includes sexism, homophobia and misogyny.

Racism must also be consistently added to the conversation and analysis (Walker \& Melton, 2015). The superfecta of hostilitysexism, homophobia, misogyny, and racism — provide a rich lens by which to examine women's experiences. In other words, coaching experiences are not the same for all women. Intersectionality impacts the psychosocial and occupational outcomes of some women and their career trajectories more acutely than others; however, without more research focused on women who possess multiple and systemic marginalization, we do not know enough about how and to what degree.

Next, researchers need to continue to focus on gendered power at the organizational and socio-cultural levels. Krahn (2019) provides a tool-feminist political economy theory-through which we can examine structural practices that have supported the current levels of women in coaching roles, and how policies reflect the institutions and leaders who produce them. By calling out such policies and the hegemonic masculine ideology they reinforce, we can expose the "hidden femininity" that impedes women in coaching (Clarkson, Cox, \& Thelwell, 2019).

Moving from research to programming and education, Larsen and Clayton (2019) speak to pathways for coaches. They call attention to the fact that women coaches have lower returns on social capital than male coaches. This is a space for action and improvement. Quality role models and mentors exist and more can be developed (Clarkson, Cox, \& Thelwell, 2019; Wasend \& LaVoi, 2019). And, as one of Banwell et al.'s participants highlighted, sponsorship is critical too as it is more about

“... connecting people, opening doors for people, making sure they know about positions that are available, putting them in the right place at the right time helping them strategically think about how they can fit a niche role that is going to be needed in the future." (Banwell, Kerr, \& Stirling, 2019, p. 132)

We cannot expect that individuals are going to have the skills, time, and motivation to mentor and/or sponsor without structures of support. Just as Allen and Reid (2019) advocated for scaffolding to develop women coaches, mentors and sponsors need scaffolding as well. The gender allies referenced earlier-and, in particular, male allies - are essential if the system is going to change. Gender allies, both internal to sport organizations and external, can be developed to call out the embedded beliefs and practices that disadvantage women coaches, and can support women coaches throughout their careers with both professional and psychosocial guidance (Banwell, Kerr, \& Stirling, 2019). Organizations need to invest resources and incentivize mentors and sponsors in order for increased returns on social capital to occur; potential incentivization includes:

- Paying them

- Releasing them from other responsibilities

- Being creative

- Taking the lead on mentorship and sponsorship

- Utilizing evidence-based best practices
However, the responsibility to find and secure sponsors and seek out allies should not be the sole responsibility of women coaches (Banwell, Kerr, \& Stirling, 2019; Clarkson, Cox, \& Thelwell, 2019). Organizations need to have leadership buy-in from the top, create a culture and vision where women leaders are not only valued and supported but also nurtured and appreciated, and back it up with resource allocation. By explicitly demonstrating that women coaches have value, and, in turn, those who support them have value, the organizational climate for women coaches will change.

Culver et al. (2019) provides another programming, or educational, pathway to change. The coaches in her article identified that they valued more self-directed education as opposed to being the passive recipients of coaching instruction. In order to maximize their zone of proximal development, resulting in increased confidence and quality of coaching, the coaches had to be actively engaged in the teaching and learning process. Communities of practice should be enacted more-both for women coaches, and as a means to develop allies like those sport leaders in Alberta. Key aspects to consider with communities of practice include the more authentic formation of groups based on participant needs, relationship-building as a result of regular interactions, and, again, dedicated resources in the form of time, space, and pay, particularly for the organizer of the community of practice. As Culver et al. found, social learning interventions are both self-directed and active. Members co-create knowledge and additional or alternative forums and ways to convene to advance the community's goals.

The common themes across the work in the special issue, and with scholars who have studied women in coaching, include educational programing that scaffolds inclusive leadership, supportive peer relationships, supportive relationships with mentors, sponsors, and organizational leaders, and visible pathways for career development (Culver et al., 2019; Larsen \& Clayton, 2019; Norman et al., 2018). In isolation, educational programs that accomplish any of these aspects can make some impact, but where all are present, structural change can occur. As Clarkson et al. (2019) and Banwell et al. (2019) pointed out, we need targeted approaches that are context-specific. Women are not a monolithic group and exist in various contexts. A "one size fits all" solution will not change the system. As stated prior, much is known about the barriers women coaches experience. Future research should focus on how to help and support women coaches within various standpoints and identity locations to thrive and survive. Supportand solution-focused research-is the next focus.

\section{For Sport Researchers}

In order to activate a change agenda, consider lending your voice in the spaces in which you operate. We've pointed to where there are gaps in the literature, and we've identified the barriers for most women coaches. So, we need researchers to lend their voices to investigating structural level change and bring examples of successful educational programming to light. Future inquiries should illuminate and focus on populations of women rarely included in research to date. For example, what are the experiences of women coaches with marginalized social identities and how do the theories and methods we use incorporate or exclude them? And, how are we examining the organizational and socio-cultural levels of the EIM? We need to focus on change at these under-examined levels.

Even after reading the manuscripts in this special issue, as researchers, questions still haunt us such as: What is at stake for women in coaching when they are in subordinate positions in relationships with significant sport others (e.g., head coaches, administrators, athletes' parents, etc.) who devalue and abuse them? How 
did the systemic oppression they suffer become systemic? What happens to a female coach's sense of self when a trusted authority figure is also an oppressor? And to what extent is the sport system that they operate in privileging coach performance above coach wellbeing? Like Fisher and Anders (2019), we also think about what is at stake if we do not respond as researchers to these issues.

\section{For Sport Leaders and Administrators}

Sport organization leaders, interrogate your policies. Do your policies reflect the voices of women coaches? And, do the policies practically inform the development of women coaches? Bottom line, are policies explicitly addressing organizational level barriers for women in coaching? Next, consider how your policies are being operationalized and how supervisors are being held accountable or incentivized to enact them. And, finally, how is your organization evaluating the effectiveness of policies and programming focused on women in coaching? If you need guidance for recruiting, hiring and retaining women coaches, see LaVoi and Wasend's 2018 report on this topic that was derived from interviewing individuals who value and support women.

In addition, as Fisher and Anders (2019) have noted, cultural sport psychology (CSP) scholars push us to engage in self-reflection and reflexivity in our professional lives, reminding us that all of us are subject to the effects of hegemony as well as majoritarian narratives (Solorzano \& Yosso, 2002). Learning to first interrogate and then resist these narratives in our own work - and in particular, as they relate to women in sport coaching-demands commitment and action. As beginning questions, we could ask ourselves: (a) How do we interrogate the effects of sexism, racism, patriarchy, cis-heteronormativity, and ableism in our own belief systems and acknowledge our complicity? (b) How do we practice aligning our values and moral commitments across our personal and then professional lives? (c) To what extent are we privileging coach performance ahead of coach well-being? and (d) How could we cultivate a commitment to women in coaching that starts with an awareness of the intersections of interpersonal and institutional power in their lives and demands incisive examinations of its deployment (Fisher \& Anders, 2019)?

\section{For Mentors, Sponsors, Coach Educators, and Allies}

Especially men with positional power, commit yourselves to learning more about the embedded sexism and gender bias in sport coaching. Then, explicitly call it out. Call it out to women coaches. Build their confidence by helping them see the bias in the system in which they work. Help them know they are not alone. The lack of women coaches is not the issue; it is a reflection of the issue. Call out embedded sexism in the organizations where you work. Be explicit and unapologetic. As Clarkson et al. (2019) stated, women coaches might be uncomfortable with positive discriminatory efforts, and so will men in positions of power. However, without discomfort being felt by the people in power within the system, the system will not change and the status quo will persist. To that end, men and women must work collaboratively together as gender allies to address systemic oppression, and "gender allyship in sport organizations offers an alternative theoretical paradigm for how sport organizations perceive and attempt to create gender equity that includes all members of the organization to be part of organizational change" (Heffernan, 2018, p. 122).

\section{For Women Coaches}

Support and advocate for each other. When given the opportunity to participate in a coaching development program-particularly women-only or women-focused - take it. Know you are not alone! Be part of the solution-resist rather than perpetuate common, false, and damaging narratives about women coaches (see LaVoi, 2016) or blame yourself or other women for the lack of women coaches. While communities of practice might not be as readily available, seek them out or ask your organization to start one for women coaches, and for male allies as well. Develop female and male mentors and sponsors. Be deliberate in identifying which men are invested in acting as allies, enlist their help, and view it as a strategic investment (Heffernan, 2018).

If there truly is a "war on women coaches" as our colleague Pat Griffin (2015) argued, everyone-all stakeholders in the sport landscape - can do something to fight the war and change the oppressive system for women coaches.

\section{Conclusion}

The articles in this special issue illuminate both challenges and best practices for women in sport coaching. Although not sought out for this purpose, they highlight the continued utility of the EIM as one way to systematically organize existing literature, stimulate dialogue, identify gaps in the literature to help researchers target inquiry, and guide decision-makers to improve the occupational experiences for women coaches. Change will not happen naturally or if only women coaches are responsible for leading the change. Decision-makers need to be held responsible as gender bias is embedded in sport organizational culture and the larger socio-cultural context; thus, change needs to occur at those levels. This special issue has identified that through research targeted at supports and conscious efforts to include diverse contexts and coaches, educational programming that scaffolds and sponsors, and policy that is specific to gender and measurable outcomes, change is possible not only in the numbers of women coaching, but also in the quality of their experiences. Both types of change are necessary moving forward.

\section{References}

Allen, J. B., \& Reid, C. (2019). Scaffolding women coaches' development: A program to build coaches' competence and confidence. Women in Sport and Physical Activity Journal, 27, 101-109.

Banwell, J., Kerr, G., \& Stirling, A. (2019). Key considerations for advancing women in coaching. Women in Sport and Physical Activity Journal, 27, 128-135.

Burton, L.J., \& LaVoi, N.M. (2016). An ecological/multisystem approach to understanding and examining women coaches. In N.M. LaVoi (Ed.), Women in Sports Coaching (pp. 49-62). London, UK: Routledge.

Clarkson, B.G., Cox, E., \& Thelwell, R.C. (2019). Negotiating gender in the English football workplace: Composite vignettes of women head coaches' experiences. Women in Sport and Physical Activity Journal, 27, 73-84.

Culver, D.M., Kraft, E., Din, C., \& Cayer, I. (2019). The Alberta Women in Sport Leadership Project: A social learning intervention for gender equity and leadership development. Women in Sport and Physical Activity Journal, 27, 110-117.

Cunningham, G.B., Ahn, N.A., Anderson, A.J., \& Dixon, M.A. (2019). Gender, coaching, and occupational turnover. Women in Sport and Physical Activity Journal, 27, 63-72. 
Fink, J.S. (2016). Hiding in plain sight: The embedded nature of sexism in sport. Journal of Sport Management, 30, 1-7. doi:10.1123/jsm.20150278

Fisher, L.A., \& Anders, A.D. (2019). Engaging with cultural sport psychology to explore systemic sexual exploitation in USA gymnastics: A call to commitments. Journal of Applied Sport Psychology, 1-17. doi:10.1080/10413200.2018.1564944

Griffin, P. (2015, January). College athletics' war on women coaches. Retrieved from http://bit.ly/2YnbTCf

Heffernan, C.D. (2018). Gender allyship: Considering the role of men in addressing the gender-leadership gap in sport organizations (Unpublished doctoral dissertation). University of Minnesota, Minneapolis, MN.

Kane, M.J., \& LaVoi, N.M. (2018). An examination of athletic administrators' perceptions regarding the absence of female head coaches in women's intercollegiate sports. Women in Sport and Physical Activity, 26, 3-11. doi: 10.1123/wspaj.2016-0031

Krahn, A.N. (2019). Sport policy praxis: Examining how Canadian sport policy practically advances the careers of nascent female coaches. Women in Sport and Physical Activity Journal, 27, 118-127.

Larsen, K. L., \& Clayton, C. J. (2019). Career pathways to NCAA Division I women's basketball head coach positions: Do race and gender matter? Women in Sport and Physical Activity Journal, 27, 94-100.

LaVoi, N.M. (2016). A framework to understand experiences of women coaches around the globe: The Ecological-Intersectional Model. In N.M. LaVoi (Ed.), Women in sports coaching (pp. 27-48). New York, NY: Routledge.

LaVoi, N.M., \& Dutove, J.K. (2012). Barriers and supports for female coaches: An ecological model. Sports Coaching Review 1(1), 17-37. doi:10.1080/21640629.2012.695891
LaVoi, N.M., \& Wasend, M. (2018). Best Practices for Hiring, Recruiting and Retaining Women College Coaches: A Study of Athletic Directors at $A$ and $B$ Grade Institutions. Minneapolis, MN: Tucker Center for Research on Girls \& Women in Sport. Retrieved from http://www. cehd.umn.edu/tuckercenter/library/docs/research/AD-Report-BestPractices.pdf

Madsen, R.M., \& McGarry, J.E. (2016). "Dads play basketball and moms go shopping!" Social role theory and the preference for male coaches. Journal of Contemporary Athletics, 10(4), 277-292.

Norman, L., Rankin-Wright, A.J., \& Allison, W. (2018). "It's a concrete ceiling; It's not even glass": Understanding tenets of organizational culture that supports the progression of women as coaches and coach developers. Journal of Sport and Social Issues, 42(5), 393-414. doi:10.1177/0193723518790086

Shaw, S., \& Frisby, W. (2006). Can gender equity be more equitable? Promoting an alternative frame for sport management research, education, and practice. Journal of Sport Management, 20(4), 483-509. doi:10.1123/jsm.20.4.483

Solórzano, D.G., \& Yosso, T.J. (2002). Critical race methodology: Counterstorytelling as an analytical framework for education research. Qualitative Inquiry, 8(1), 23-44. doi:10.1177/107780040200800103

Walker, N.A., \& Melton, E.N. (2015). The tipping point: The intersection of race, gender, and sexual orientation in intercollegiate sports. Journal of Sport Management, 29(3), 257-271. doi:10.1123/jsm. 2013-0079

Wasend, M., \& LaVoi, N.M. (2019). Are women coached by women more likely to become sport coaches? Head coach gender and female collegiate athletes' entry into the coaching profession. Women in Sport and Physical Activity Journal, 27, 85-93. 